\title{
Cross-layer transmit power and beacon rate adaptation for VANETs
}

DOI:

$10.1145 / 2512921.2518070$

Link to publication record in Manchester Research Explorer

\section{Citation for published version (APA):}

Sander Frigau, M. (2013). Cross-layer transmit power and beacon rate adaptation for VANETs. In DIVANet 2013 Proceedings of the 3rd ACM International Symposium on Design and Analysis of Intelligent Vehicular Networks and Applications, Co-located with ACM MSWiM 2013/DIVANet - Proc. ACM Int. Symp. Des. Anal. Intelligent Veh. Networks Appl., Co-located ACM MSWiM (pp. 129-136). Association for Computing Machinery. https://doi.org/10.1145/2512921.2518070

\section{Published in:}

DIVANet 2013 - Proceedings of the 3rd ACM International Symposium on Design and Analysis of Intelligent Vehicular Networks and Applications, Co-located with ACM MSWiM 2013|DIVANet - Proc. ACM Int. Symp. Des. Anal. Intelligent Veh. Networks Appl., Co-located ACM MSWiM

\section{Citing this paper}

Please note that where the full-text provided on Manchester Research Explorer is the Author Accepted Manuscript or Proof version this may differ from the final Published version. If citing, it is advised that you check and use the publisher's definitive version.

\section{General rights}

Copyright and moral rights for the publications made accessible in the Research Explorer are retained by the authors and/or other copyright owners and it is a condition of accessing publications that users recognise and abide by the legal requirements associated with these rights.

\section{Takedown policy}

If you believe that this document breaches copyright please refer to the University of Manchester's Takedown Procedures [http://man.ac.uk/04Y6Bo] or contact uml.scholarlycommunications@manchester.ac.uk providing relevant details, so we can investigate your claim.

\section{OPEN ACCESS}




\title{
Cross-layer Transmit Power and Beacon Rate Adaptation for VANETs
}

\author{
Matthias Sander Frigau \\ School of Computer Science \\ University of Manchester \\ Manchester M13 9PL, United Kingdom \\ msanderfrigau@cs.man.ac.uk
}

\begin{abstract}
The key benefit of VANETs comes in the form of safety applications. Broadcast is the predominant form of communication for safety messages. However, due to the fast changes occurring in the network topology (sparse, dense, congested), vehicles within a VANET may face many disturbances such as collisions which can cause performance degradations. Thus it becomes hard to maintain an efficient channel utilization and so maximize the network capacity. In this paper, the idea is to take into account the dynamically changing topology of a VANET (local traffic density) and have each vehicle able to dynamically adapt its PHY QoSparameter (Transmit Power) according to its fast changing channel conditions, network load, and link quantities of upper-layers. The proposed mechanism, called Transmit Power Adaptation (TPA) relies on channel estimate at PHY layer and uses feedback from an adaptive beaconing system (also presented) which builds the local view of a vehicle at the network layer. We have evaluated the performance of TPA through simulation with ns-3 simulator. Results show that TPA clearly outperforms the default 802.11 broadcasting mechanism in terms of network capacity. TPA also outperforms a similar adaptive technique not based on channel estimate in terms of network capacity for three scenarios.
\end{abstract}

\section{Categories and Subject Descriptors}

C.2.1 [Computer-Communication Networks]: Network Architecture and Design - Network topology, Wireless communication; C.2.3 [Computer-Communication Networks] Network Operations - Network monitoring

\section{General Terms}

Algorithms, Design, Performance

\section{Keywords}

Vehicular networks, cross-layering, beacon fairness, network metrics, context-awareness

Permission to make digital or hard copies of all or part of this work for personal or classroom use is granted without fee provided that copies are not made or distributed for profit or commercial advantage and that copies bear this notice and the full citation on the first page. Copyrights for components of this work owned by others than ACM must be honored. Abstracting with credit is permitted. To copy otherwise, or republish, to post on servers or to redistribute to lists, requires prior specific permission and/or a fee. Request permissions from permissions@acm.org.

DIVANet'13, November 3-8, 2013, Barcelona, Spain

Copyright 2013 ACM 978-1-4503-2359-8/13/11 ...\$15.00.

http://dx.doi.org/10.1145/2512921.2518070.

\section{INTRODUCTION}

The last decade has witnessed a growing interest in Vehicular Ad-hoc Networks (VANETs) which aim at building self-configuring networks made of vehicles on the road. By enabling communication between vehicles, new Intelligent Transportation System (TS) applications will improve road traffic safety and driving experience. In a VANET, context-aware information is related to the vehicle's position, speed, road topology, space-time characteristics of the communication graph and environmental conditions. However, such information is short-term-consistent due to the high dynamics involved in a VANET. A VANET can have many different configurations: it can be a dense network in a limited geographic area with few interferences or it can be a sparse network in a large city environment with lots of interferences. For each configuration, a vehicle needs to dynamically self-adapt its network parameters according to its context changes. So, a VANET must work properly in a wide range of conditions: it must handle different vehicular densities, road topologies, and channel conditions. A vehicle in a VANET must ensure a minimal quality of service regardless of the conditions in the environment. Hence, a vehicle should be able to dynamically adjust its fixed QoS parameters by estimating the probability of successfully delivered packets (PRR) [16] based on its local traffic density and metrics capturing network conditions on the road. Thus, the fixed QoS parameter Transmit Power needs to be adjusted according to the degree of uncertainty of the channel and the environment.

Link quantitities such as the link lifetime (which depicts the time two nodes can spend communicating before they are physically disconnected) and the average distance to neighbours (can be seen as the inter-vehicle distance) are important metrics that describe the VANET communication graph characteristics. As most of the links are short-lived in a VANET, a common strategy to increase the lifetime of a link is by increasing the Transmit Power. However using a higher power level in a dense traffic environment may result in a large amount of interferences.

Although Transmit Power Control in VANETs has been widely studied in the literature [11], [12], [13], related work has not investigated the effect of using link lifetime nor the average distance to neighbours (which helps to detect traffic congestion) to other vehicles in the transmission range. In this paper, the use of such metrics when transmitting 1hop broadcast packets is considered to improve the network capacity. 
The main contribution of this paper is the introduction of a new joint approach in order to dynamically adapt the PHY Transmit Power and the Beaconing Rate at L3 layer while monitoring specific VANET metrics and channel qualities.

The structure of this paper is as follows: in the next section, we present the relevant related work. In Section 3, assumptions for our systemthe are presented, then a presentation of the Transmit Power Adaptation algorithm is given. In Section 4 we outline our simulation settings, the methodology used, and according to our evaluation criteria we have plotted the results for the default scheme, Rawat et al. scheme [8] and TPA scheme for three scenarios. Finally Section 5 presents our conclusion and future perspectives based on this study.

\section{MOTIVATION AND ASSUMPTIONS}

Applications in a VANET are mainly related to driving safety and are of high interest in research studies. These safety-related applications are low-delay constrained and involve most of the time one-hop broadcast communication. Two types of safety-related information can be defined: beacon messages and event-driven messages.

Beacons: When assuming a geographic routing protocol in use within the network, there is often a beaconing system providing periodic status information about the surrounding environment. An equivalent to beacons is used within the WAVE standard: WAVE Short Messages (WSMs). In this paper, we use the WAVE Short Messages (WSMs) as provided by the IEEE 1609.4 standard but we will refer throughout this paper to beacons. Each vehicle periodically broadcasts beacons (small packets) which contain mobility information (speed, angle, position) or other context-aware information.

Event-driven messages: Each vehicle broadcasts its information to its reachable neighbours; this aggregated information is used by receivers to determine if there is a need for a safety response (slowing down, changing lanes). This kind of safety-related broadcast is used to warn vehicles of a potential emergency (event-driven messages) [13]. However, for dense traffic conditions, periodic one-hop broadcast can quickly overload the radio channel and cause a massive amount of packet collisions. If these collisions are not detected, the network can quickly become congested. As a result, the packet delivery ratio drops dramatically. In this context, where varying densities are expected and given the broadcast nature of communications, maximizing the resources (especially the network capacity - i.e. throughput) is a major challenge. Hence we propose a strategy which aims at adapting dynamically the PHY Transmit Power by monitoring the channel conditions (Packet Reception Rate) and the channel load (for event-driven messages and beacons) so we do not wait for performance degradations. The transmit power level is measured in $\mathrm{dB}$ and determines the quality of the signal received at the receiver and determines the transmission range $r$. In theory, and according to the observations in [5], adjusting a fixed parameter such as the Transmit Power at PHY layer can significantly impact the Network layer:

1. Decreasing the power levels increases the network capacity and reduces the average contention at MAC layer.
2. When the traffic load in the network is high, a lower power level gives lower end-to-end delay.

3. Transmit Power gives the routing protocol the opportunity to determine the optimal next hop or the intended receiver so the network-layer allows for global optimization.

4. Intended receiver is determined by the network layer (routing protocol neighbours table) consequently the Transmit Power Adaptation is not determined at MAC. MAC does only a local optimization.

Thus, the MAC approach to transmit power adaptation only does a local optimization, whereas network-layer transmit power adaptation is capable of a global optimization. The transmit power adaptation problem is a cross-layer problem which affects PHY and upper-layers of the protocol stack.

\section{RELATED WORK}

In [7] after a deep analysis of spatio-temporal characteristics of the VANET's communication graph, the authors state that adjustments of the data rate and transmission power are mandatory. The Fcar protocol presented in [14] makes routing decision based on two metrics/quantities (route lifetime and percentage of same-directional vehicles). Those two metrics are given as inputs to a rule-based fuzzy controller system which then evaluates the route selection probability (based on AODV). The set of rules of the fuzzy controller is not precise as different rules can lead to the same route probability. Plus, the density in their study has not been used which has been proved to be of great importance when dynamically adapting a network parameter. Another study [10] shows that the network connectivity is affected by the network density so they dynamically adjust the Backoff time 802.11p MIB parameter according to the traffic density. With the help of a fuzzy analyzer the authors insert the measured density at the network layer and the backoff time. The output is an adapted backoff. However, no transmit power adaptation has been included.

The most related study to our work has been carried out by Yang et al. [16]. Their proposed method optimizes the reception rate and channel utilization of periodical broadcast messages. Each vehicle adjusts its transmission power according to two parameters: a predefined reception rate threshold and a predefined traffic load (number of neighbours) threshold. However they do not take into consideration, when estimating the channel conditions, the relative distance between sender and receiver, and, they do not adapt the beaconing data rate. Torrent-Moreno et al. investigated the low reception rate problem in broadcast communications [11] [13]. The Transmit Power and Beaconing data rate is adjusted in order to achieve a certain probability of reception at a specific distance but the link lifetime is not considered.

More recently, Rawat et al. proposed a Joint Adaptation Algorithm [8] which estimates vehicle density via the exchange of DSRC status messages (broadcasted 10 times per second). They have considered the adaptation of the Transmit Power, not based on channel conditions but on an estimated Transmission Range (estimation based on road segment length). 


\section{SYSTEM MODEL}

This section contains the definitions of the metrics used in this study. This section then describes how the channel conditions are estimated based on local density, upper-layer link quantities and beacon load. Then we give assumptions and detail about the algorithm. The algorithm combines the advantages of dynamic self-adaptation of Transmit Power at PHY layer as a function of vehicle density, and link quantities (i.e. link lifetime, average distance to neighbours) with dynamic adaptation of beacon sending rate.

\subsubsection{Communication Model}

The VANET communication graph is represented as an undirected graph $G(t)$ at time $t . V(t)=\left\{u_{i}\right\}$ the set of vertices, represents the vehicles in the network where $V \in \mathbb{R}^{+}$, and $E(t)=\left\{e_{i j}\right\}$ the set of edges, represents the direct communication links. An edge $e_{i j}(t)$ exists iif $u_{i}$ can communicate with $u_{j}$ at time $t$, with $i \neq j$. Each vehicle $u_{i}$ has a list of vehicles within its transmission range. The list of neighbours is formally defined as: $\left\{u_{j} \mid \exists e_{i j}(t) \in E(t)\right\}$ and the number of neighbours is defined as $k_{i}(t)=\|\left\{u_{j} \mid \exists e_{i j}(t) \in\right.$ $E(t)\} \|$.

The list of neighbours, which is obtained by building a neighbours table based on the received beacons, is as follows : each entry (neighbour) gives us location information, power level information, signal-to-noise plus interference ratio, link lifetime. 2-hop neighbours are not considered as we do not focus on relaying unicast traffic.

The link lifetime - $L L$ is the time between the instant at which a vehicle enters another's vehicle transmission range and the instant at which the physical connection is lost. If we consider vehicle $u_{i}$ and vehicle $u_{j}$ being in the same transmission range, the duration is formally defined as $l_{i j}$ if $\exists e_{i j}$. The link lifetime is calculated with the help of a formula using mainly the euclidean coordinates :

$$
L L=\frac{-(a b+c d)+\sqrt{\left(a^{2}+c^{2}\right) r^{2}-(a d-b c)^{2}}}{a^{2}+c^{2}},
$$

where $r$ is the transmission range and vehicles $i$ and $j$ have respectively the mobility vectors $\left(x_{i}, y_{i}\right), \theta_{i}$ and $\left(x_{j}, y_{j}\right), \theta_{j}$ where:

$a=v_{i} \cos \theta_{i}-v_{j} \cos \theta_{j}$

$b=x_{i}-x_{j}$

$c=v_{i} \sin \theta_{i}-v_{j} \sin \theta_{j}$

$d=y_{i}-y_{j}$

\subsection{Beaconing: Neighbours Status and Local Traffic Density}

In vehicular networks, each vehicle already possesses context information about the environment, in the form of the location and speed of itself and its neighbours [9]. Knowledge about the environment is often gained via a beaconing system. Each vehicle periodically sends beacons to its reachable one-hop neighbours. Those beacons most of the time contain mobility information such as the Euclidean coordinates of the vehicle, its speed, its angle, its nodal degree or other network-related information. Hence each vehicle has a one-hop local view of its environment. Each vehicle has knowledge of its neighbours' status through a neighbours table. Therefore it is easy to compute the local traffic density. The neighbours' table is updated according to the sending rate of beacons. If the beacon interval is set to 1 second, hence we will count, every 1 second, the number of
MAC entries (which is our Local Traffic Density). We note that our way to collect the local traffic density is different from Rawat and al. [8]. They use a similar estimation technique as [16] by counting the number of sequence numbers in DSRC status messages (10 packets / second). Our approach generates less overhead as we use cross-layering to pass the Local Traffic Density to the PHY layer. Our beacon rate is initialized to 1 beacon broadcast per second and is then adapted according to the beacon load and current sensed conditions.

Beaconing is important because:

1. it conveys periodic messages with context-aware information,

2. its beacons are preventive messages in terms of safety,

3. it gives an estimate of the state about the vehicles in the neighbourhood,

4. it is fundamental to geographic routing protocol or to non safety applications / monitoring applications,

5. it improves driver awareness of the surrounding environment.

This allows our adaptation mechanism to be correlated with a routing protocol so that we could, as a further study, analyze how the adaptation affects the routing protocol.

It is possible to enhance the beaconing system so that each vehicle can gain more accurate information and adapt its fixed QoS-related parameters by estimating new metrics. This can be achieved by using cross-layering to retrieve other layer information or to combine different metrics from different layers. MOPR [6] uses movement information available at the MAC layer to predict the future positions of relay vehicles and calculate the Link Stability based on which the forwarding selection will be performed. In CLWPR [4] the distance on the road is used as a metric (curvemetric distance), as well as a link quality estimation using the Signal to Noise-plus-Interference Ratio (SNIR) information and MAC Frame Error rate and the vehicle's utilization (queue status) to balance the traffic load.

\subsubsection{Beaconing Load and MBL upper-bound}

It is expected that the VANET needs a tradeoff between driver's awareness and beacon load (beacon fairness). Beacon messages are relevant for vehicles in the close neighbourhood and this relevance decreases as the distance increases. However, a high number of beacons has to be avoided as it can result in a high number of collisions. So we have implemented an algorithm based on Torrent-Moreno et al. work [11] in order to properly manage the resource allocation for event-driven messages. We have to maintain a sufficient capacity for beacon load without exceeding a proper and fixed Maximum Beacon Load (MBL) and at the same time we need enough bandwidth for event-driven messages. Our Adaptive Beaconing algorithm is depicted in Algorithm 2. In order to increase the beacon load we properly adjust the beacon interval (fixed at 1 sec.) and the Transmit Power of beacons depending on safety distance (in our case, it is the aggregated average distance to neighbours) and the aggregated average link lifetime of neighbours. As the distance is critical for beacons, close distances need to be considered. Thus, when a vehicle $u_{i}$ senses a situation where vehicles in 


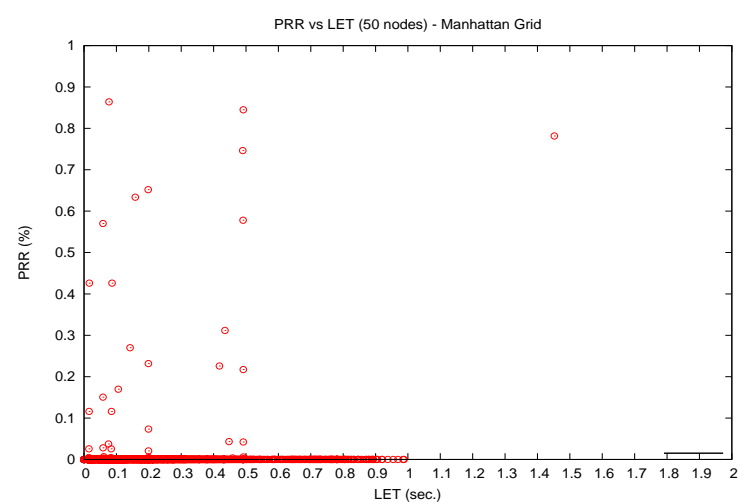

Figure 1: PRR vs LET for 50 nodes - Manhattan Grid

its neighbourhood are below a specific safety distance (intervehichle distance) $d_{s}$ it means that it is more likely that an unsafe proximity event will occur (such as congestion) and then the fairness of beacons becomes crucial. Hence, for safety reasons, we need to provide our neighbours with more accurate informations and we increase the beacon rate (if we are below MBL). However, if the average lifetime of the neighbours links is lower than an adapted threshold (based on lifetime history), we decrease the beacon rate. Our beacons include the position (Euclidean coordinates), the velocity vector, and the transmit power. With the help of the mobility and velocity vectors, we can easily compute the link lifetime of any vehicle in the neighbourhood. Hence with the help of the link lifetime prediction formula we can detect fast moving conditions by sensing the stability of the links. Through empirical evaluations, we observed that the majority of vehicles' link lifetime varies between 0sec. and 2sec. in urban scenarios. This is depicted in Fig. 1.

\subsection{Transmit Power Adaptation}

\subsubsection{Algorithm}

We assume that the vehicles start running the algorithm as soon as they receive beacons or event-based messages. This algorithm is performed every $\tau=1$ second. We have designed an adaptive strategy (the algorithm is shown in Algorithm 1) which exploits the sensed traffic load in the neighbourhood of one vehicle and adapt its Transmit Power (dB) according to a specific traffic density threshold $\Delta$, a Packet Reception Rate (PRR) expression and beacon load. The mathematical formulation of this PRR equation is based on a stochastic analysis and is discussed in [16]. The local traffic density $n$ is an important factor as it is observed in [10]. The network connectivity is highly dynamic and is affected by the vehicle's density. Let us define our PRR formula: $T_{P H Y}$ is the duration of the PLCP (Physical Layer Convergence Protocol) headers and preamble. $T_{M A C}$ is the duration of the MAC headers; $T_{D I F S}$ relates to the duration a vehicle needs to wait after sensing the channel is idle and before transmitting. If we define the packet length as $S$ and the datarate as $R$ the duration of a frame payload is equal to $S / R$. We assume that all slots have the same duration $\sigma=S / R$. The duration of a successful transmission attempt is: $T_{\text {succ }}=T_{P H Y}+T_{M A C}+T_{D I F S}+S / R$. Since the contention process can be represented as a slotted discrete-time process, the length of a successful transmission attempt, in number of slots, is:

$$
n_{\text {slots }}=\frac{T_{\text {succ }}}{\sigma}=\frac{T_{\text {succ }} * R}{S}
$$

with $\sigma$ being the duration of a time slot. Now, if a vehicle wants to transmit in a time slot, the attempt probability is:

$$
P_{\text {transmit }}=\frac{1}{n_{\text {slots }}}=\frac{S}{T_{\text {succ }} * R}
$$

Thus, we can define our $P R R$ formula as the probability that a vehicle successfully receives a packet with $n$ neighbours located in its transmission range:

$$
P R R=\left(1-\frac{S}{T_{\text {succ }} * R}\right)^{n}
$$

with $S$ being the packet size and $R$ the data rate.

Xue et al. [15] in their study showed that each vehicle should be connected to $\Theta(\log n)$ nearest neighbours. If a vehicle is connected to more than $5.1774 * \log (n)$ nearest neighbours then the network is asymptotically connected. We will take this value as a reference threshold named $\Delta$ and $\Delta=5.1774 * \log (n)$ with $n$ being the total number of neighbours randomly placed in a map. Hence, if the number of neighbours for a vehicle is below $\Delta$ threshold it means that this vehicle is in a sparse topology and less collisions will occur as the MAC channel is not fully utilized. In this case we increase the Transmit Power by one power unit. Otherwise, if $n$ is above $\Delta$, we do not increase the Transmit Power immediately.

Instead, if $P R R$ is above a fixed PRR-threshold $\alpha$ we maintain PRR and make possible improvements by increasing the Transmit Power. Else, if $P R R$ is below $\alpha$ we try to improve it by decreasing the Transmit Power by one power unit and thus avoiding the possibility of encountering more collisions. Indeed, in our approach we have implemented a beaconing mechanism which periodically broadcasts small packets. Our method to estimate accurately the density $n$ for a given vehicle is to sense the number of neighbours by exploiting the beaconing mechanism. Our strategy is performed every $\tau$ (fixed to 1 ) and allows us to capture an accurate estimation of the number of vehicles that can be present within a vehicle's transmission range.

\section{EVALUATION}

In order to evaluate the delivery performance of 1-hop broadcast packets we have used the version 3.15 of the ns3 network simulator. The physical layer uses the IEEE $802.11 \mathrm{p}$ standard with a data rate of $6 \mathrm{Mbps}$. This data rate is chosen according to its low SNR value (around $4 \mathrm{~dB}$ ). Vehicles transmit packets only using the $\mathrm{CCH}$ radio channel (no channel switching). The beaconing mechanism closely follows the WAVE Short Message Protocol and has been implemented has a separated module. The main simulation settings are listed in Table 1. During simulations we observed PRR values ranging from 0.4 to 0.9 mainly. So the PRR-threshold in TPA algorithm is fixed to 0.6 and is then adapted according to PRR values history. The MBL is fixed to $40 \%$ of the total available bandwidth. This means that $40 \%$ of the channel capacity can be dedicated for the beacons. Our TPA has been implemented as a new PHY layer within ns-3 simulator. Our beacon mechanism starts with a default interval of 1 second. We have generated three different road network scenarios: 


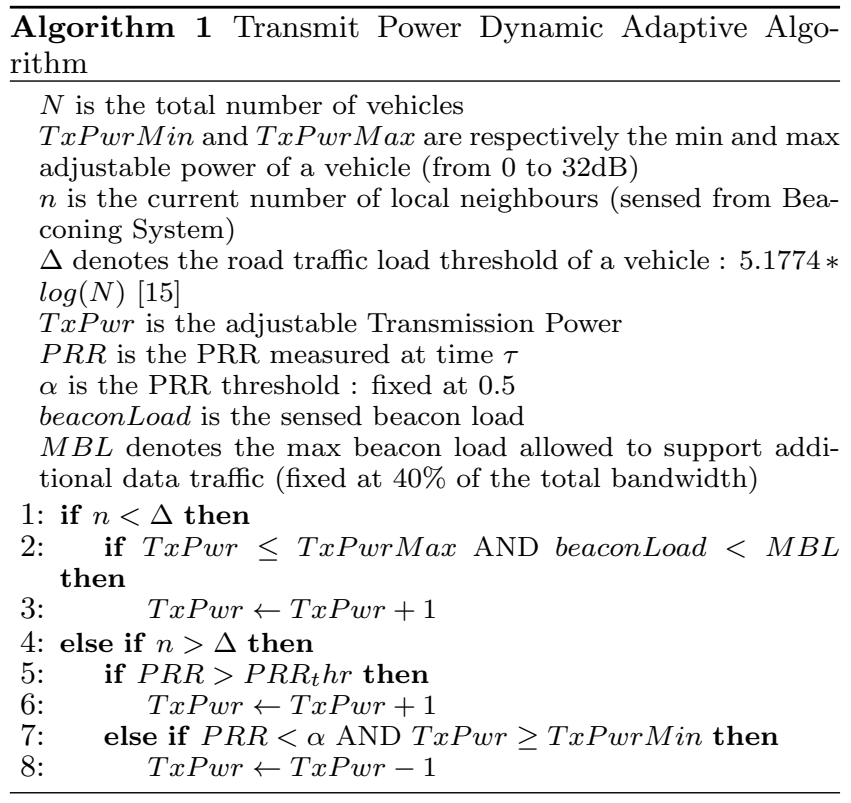

1. Scenario 1 - Manhattan Grid: this road network has been generated using the mobility generator BonnMotion [1]. The Manhattan grid is a $5 \times 5$ grid with edge length of $600 \mathrm{~m}$. The area is a $3000 \times 3000$ grid map. The average vehicle speed is $14 \mathrm{~m} / \mathrm{s}$. We have produced vehicles movements for both sparse and dense traffic densities with 50 and 150 vehicles respectively.

2. Scenario 2 - Random Voronoi Map: this scenario uses more realistic vehicular mobility traces as we apply an Intelligent Driver Model (IDM). IDM_LC (Intelligent Driver with Lane Change) considers mobility at a microscopic level thus describing perfectly car-tocar and intersection managements. We have used the tool VanetMobiSim [2]. The settings of IDM_LC are shown in Tab. 2.

3. Scenario 3 - TIGER Map: We have generated vehicular movements based on user-defined trips in order to simulate congested urban road traffic. We have used the MOVE mobility generator [3] with the road network map of the West University Place, Houston, TX, USA ( Fig. 2) which is available from the public TIGER database of the U.S. Census Bureau. The map area is $2387 \times 2373 \mathrm{~m}$.

The Two-Ray Ground propagation model is used with a path loss exponent $\alpha=2$. We vary the propagation model for the Voronoi Map scenario: the Two-Ray Ground propagation model and the Rayleigh distribution based fast fading. Rayleigh fading models the situation when there is no LOS (Line-Of-Sight). We start to broadcast beacons periodically at the start of the simulation and each vehicle starts generating broadcast traffic after 20 seconds of the simulation.
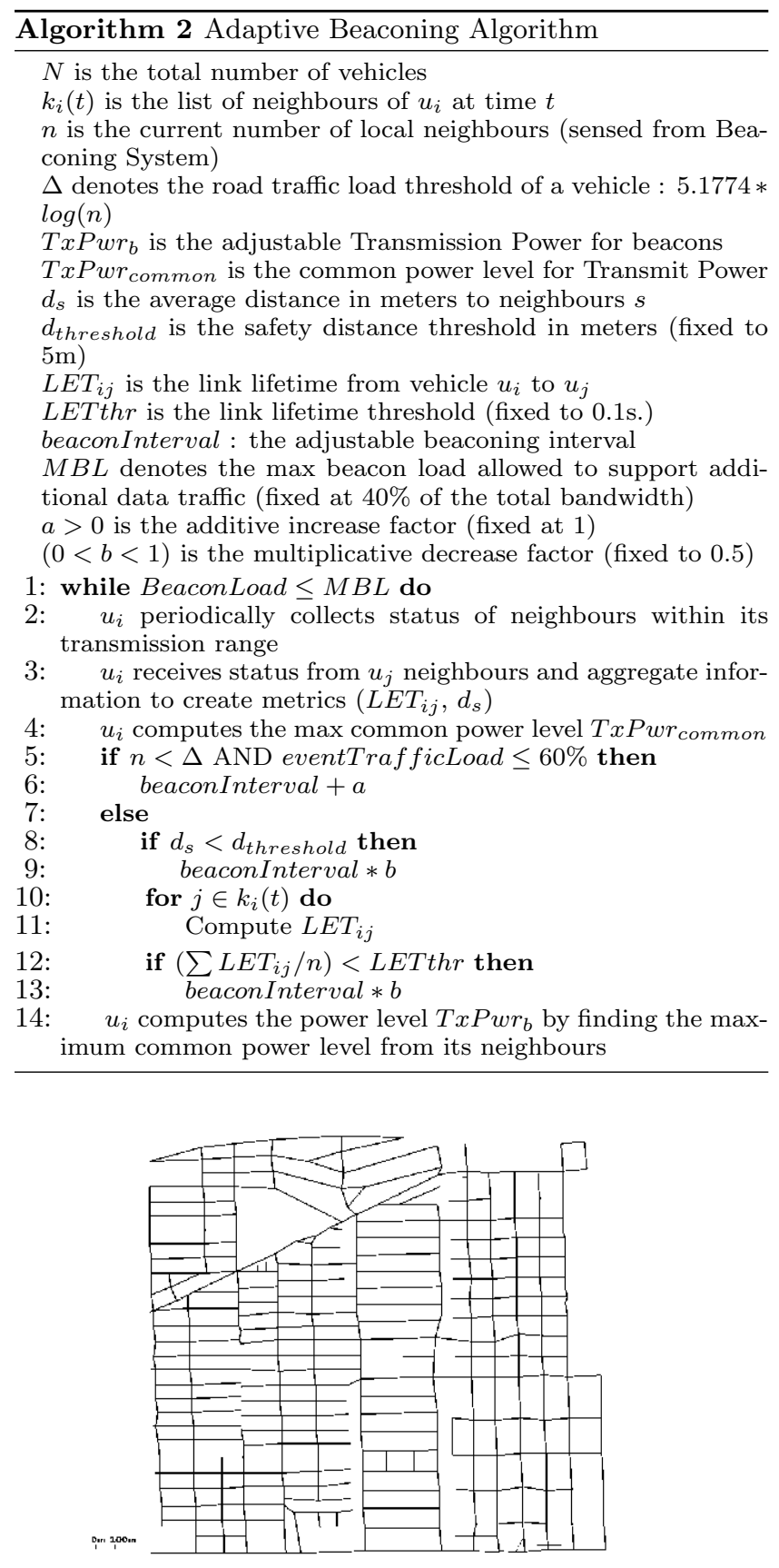

Figure 2: West University Place - Houston, TX, USA

\subsection{Results}

To evaluate the performances of our TPA algorithm we have compared it with the default 802.11 mechanism and the Rawat et al.[8] adaptive algorithm. For the latter, we picked the same parameters and values and implemented their algorithm as described in [8]. However, we note that we have not included the adaptation of the CW size for the Rawat et al.[8] adaptive algorithm. This is due to the fact that our focus is to adapt the transmit power in order to maximize the network capacity. To represent our eventdriven safety messages we broadcast packet of size 500bytes, while the beacon packet size is 100 bytes. Several other pa- 
rameters for PHY and MAC layer are detailed in the simulation settings (Tab. 1). To evaluate the effectiveness of our TPA algorithm, we have measured the throughput and the end-to-end delay. We selected a packet generation rate for event-driven messages of 10packets/s. The default scheme uses a fixed transmission range of $250 \mathrm{~m}$ and a fixed transmission power of $16 \mathrm{~dB}$. TPA and Rawat et al. algorithms vary the transmit power between 0 and $32 \mathrm{~dB}$. The results for each scenario are explained below:

\subsubsection{Scenario 1}

For the Manhattan Grid, results show the variation for the throughput and delay varying with time. In Fig. 3b the results clearly show a significant increase in the overall throughput. When vehicles start to generate event-driven safety messages (at 20sec.) the default scheme does not show any any variation in the throughput due to its fixed transmit power. Rawat et al. manage to maximize some throughput and this throughput remains constant during the 100 seconds of the simulation. As TPA does the adaptation in a different way (it uses the estimate of the channel conditions), it manages to allocate the resources more efficiently and more accurately. After around 30 seconds, our TPA algorithm starts to stabilize the total throughput consumption, which remains close to $1.5 \mathrm{Mbps}$ for the rest of the simulation time. Thus, TPA outperforms the default scheme and Rawat et al. algorithm in terms of throughput in a dense traffic density scenario (150 vehicles).

As we have not included the adaptation of the CW size, the end-to-end-delay in Fig. 3a is slightly higher than the default scheme for both algorithms. TPA does not show any improvement and shows a similar behaviour as for Rawat et al. algorithm. Those high picks in the end-to-end delay can be explained by the fact that if we adjust the transmit power we are going to be able to reach vehicles located at farther distances and thus observe an increase in the propagation delay.

\subsubsection{Scenario 2}

For the Voronoi Map scenario we have plotted results for the Two-Ray Ground propagation model. The throughput in Fig. 3d stabilizes around 1.5Mbps for TPA, and only $500 \mathrm{Kbps}$ for Rawat et al. algorithm. As shown in Fig.3c the end-to-end delay is similar for the 3 schemes until we start broadcasting traffic at 20sec. From 0 to $20 \mathrm{sec}$ the delay remains close to $250 \mathrm{msec}$ and is the same for the three schemes. After 20 seconds (when we start broadcasting event-driven messages) the delay reaches values up to $550 \mathrm{msec}$ for TPA. As we are able to increase the transmission range we are going to increase the link distance. Consequently, for the default scheme we observe a average distance to neighbours of $35 \mathrm{~m}$, for the Rawat et al. algorithm this distance is close to $150 \mathrm{~m}$ and for TPA the average distance to neighbours is close to $160 \mathrm{~m}$. Here TPA again shows a great improvement in maximizing the overall network capacity. Results for the Nakagami propagation model show that the Rawat et al. algorithm slightly outperforms TPA in between 0 and 20sec. of the inital simulation step (beacons only). But then as TPA stabilizes and start adapting the transmit power according to channel conditions, link lifetime, and beacon load, TPA starts to increase monotonely and has a higher throughput compared with other schemes.
For the end-to-end delay, we observe a similar trend for the three schemes (Fig. 3c).

\subsubsection{Scenario 3}

In this scenario, we have defined manually vehicle trips in a realistic map. The delay for the three schemes is shown in Fig. 3g. Our results indicate that there is no major variation between the default, TPA and Rawat et al. algorithm for the end-to-end delay. However, for the throughput in Fig. 3h a significant increase is observed for TPA after around 30 seconds of the simulation time. The throughput remains stable (around 2.5Mbps) for the rest of the simulation. Again, TPA shows great improvements in optimizing the network capacity.

Table 1: Simulation settings

\begin{tabular}{|c|c|c|}
\hline PHY & Frequency & $5.9 \mathrm{Ghz}$ \\
\hline & Channel Bandwidth & OFDM 10Mhz \\
\hline & Data Rate & $6 \mathrm{Mbps}$ \\
\hline & Noise Floor & $-103 \mathrm{dBm}$ \\
\hline & Energy Detection Threshold & $-85 \mathrm{~dB}$ \\
\hline & Antenna Gain & $1 \mathrm{~dB}$ \\
\hline MAC & Slot Time, $\sigma$ & $13 \mu \mathrm{s}$ \\
\hline & $C W_{\min }$ & 15 \\
\hline Network & $C W_{\max }$ & 1023 \\
\hline & Protocol & WSMP $/$ IEEE 1609.3 \\
\hline Application & Beacon Initial Interval & $1 \mathrm{~s}$ \\
\hline & Packet Generation Rate & $10 \mathrm{pkts} / \mathrm{s}$ \\
\hline & Traffic Type & UDP Broadcast \\
\hline
\end{tabular}

Table 2: Voronoi Map and IDM_LC settings

\begin{tabular}{|c|c|}
\hline Map Area & $6000 \times 6000$ \\
\hline Min Speed & $8.33 \mathrm{~m} / \mathrm{s}$ \\
\hline Max Speed & $13.89 \mathrm{~m} / \mathrm{s}$ \\
\hline Number of lanes & 2 \\
\hline Deceleration & $0.5 \mathrm{~m} / \mathrm{s}^{2}$ \\
\hline Stay Duration & $0.5 \mathrm{~m} / \mathrm{s}^{2}$ \\
\hline
\end{tabular}

\subsection{Observations}

What can be inferred from the above simulation results? From the above three scenarios, it is straightforward to infer that the association of a transmit power algorithm with an adaptive beaconing algorithm can significantly improve the network capacity. For scenario 1 and scenario 2, the network throughput is maximized up to $40 \%$ when compared to Rawat et al.[8]. Such findings make our algorithm much more suitable for VANETs than an algorithm not based on channel estimate. For TPA, the delay closely match the delay of Rawat et al.[8] algorithm. The third scenario simulates a congested area with mainly two paths that vehicles follow. We observed that in such conditions the beacon rate has a tendency to decrease as the safety distance is no more respected and the average link lifetime of neighbours drops below a specific threshold. Consequently the gain in network capacity is not as important as for the two other scenarios.

What are the main lessons learned from this study? Here, a vehicle monitors its channel conditions and its surroundings and reactively adapts some parameters to some specific changes. Hence, the road length, as suggested by Rawat 


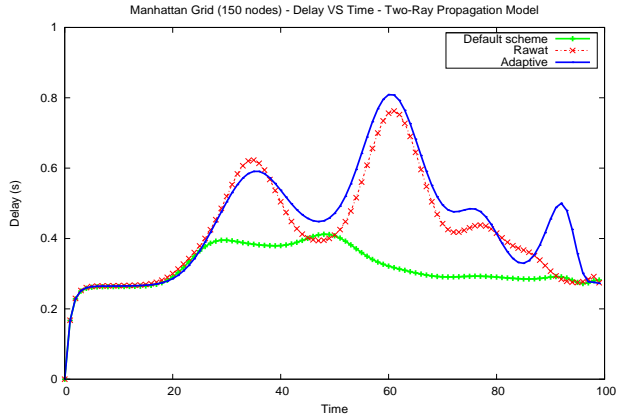

(a) Scenario 1: Manhattan Grid - Delay

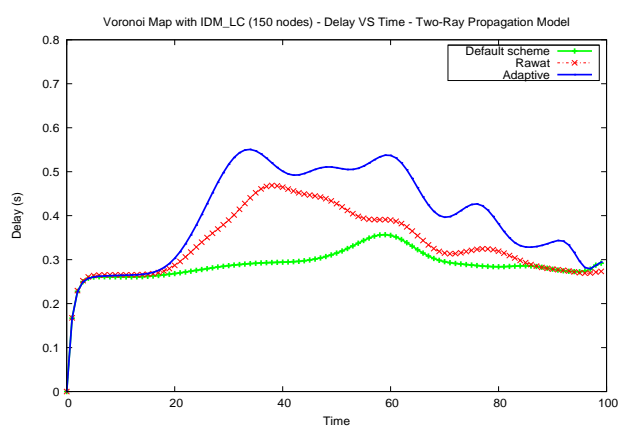

(c) Scenario 2: Voronoi Map - Delay (TwoRay)

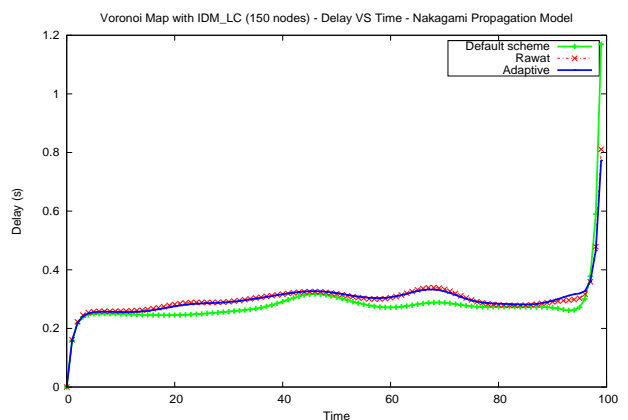

(e) Scenario 2: Voronoi Map - Delay (Nakagami)

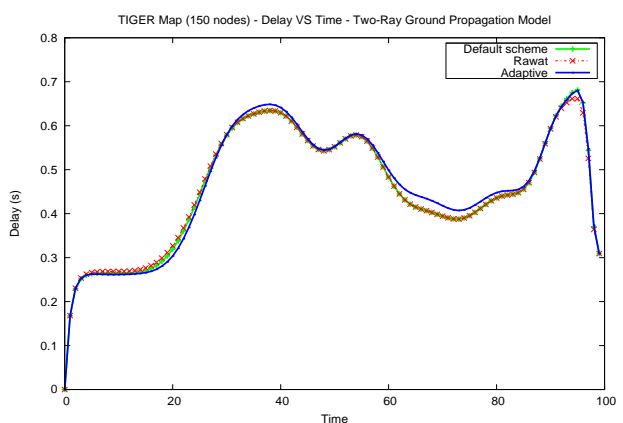

(g) Scenario 3: TIGER Map - Delay

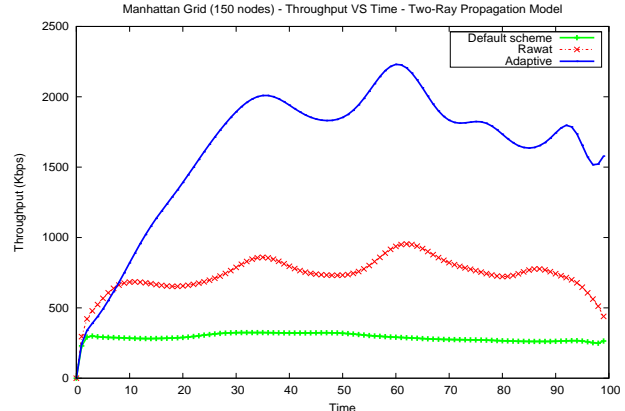

(b) Manhattan Grid - Throughput

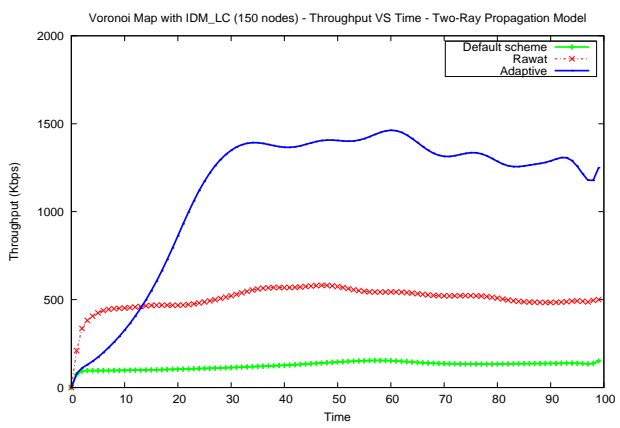

(d) Voronoi Map - Throughput (Two-Ray)

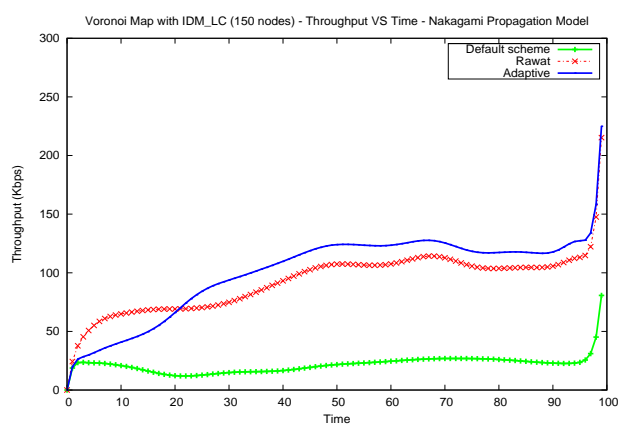

(f) Voronoi Map - Throughput (Nakagami)

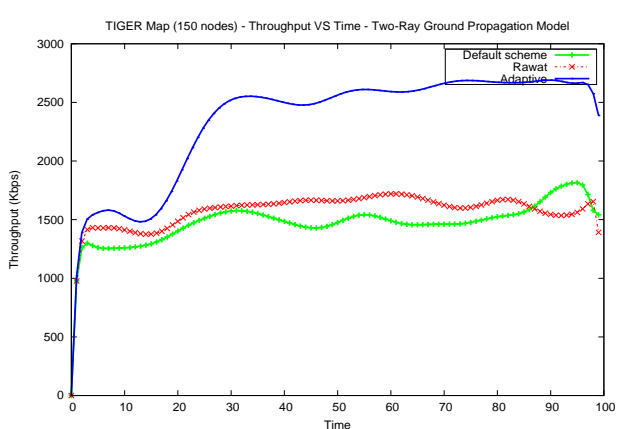

(h) TIGER Map - Throughput

Figure 3: Simulation results for the three scenarios: Manhattan Grid, Random Voronoi Map w/IDM_LC, TIGER Map 
et al.[8] is not a sufficient criterion to adapt the transmit power in a dynamic environment such as a VANET. The integration in TPA of the link lifetime, the safety distance of neighbours as well as the way we compute the packet reception rate help in maximizing the network throughput. All those metrics are computed through the use of traffic context awareness following a closed-loop approach. Each vehicle can configure its parameters based on the use of context aware information obtained though the periodic broadcast of beacons. TPA highlights the need for further research on a multi-scenario adaptive approach.

\section{CONCLUSION}

Beacon rate and transmit power adaptation represent an essential building block to ensure the stable operation of vehicular safety applications based on broadcasts. In this paper, we have presented a Cross-layer Transmit Power Adaptation for efficient broadcast communication in urban VANETs. It has been observed through simulations that we obtain better performance when we adapt the Transmit Power according to the channel conditions, the local traffic density, the beacon load, and link quantities such as link lifetime and the average distance to neighbours. Each vehicle can dynamically adjust its beacon rate and transmit power and select the appropriate transmit power of event-driven safety packets according to the changes in the local traffic density and by monitoring critical safety situations (fast changing conditions or congested conditions). The proper adaptation of the transmit power makes the wireless radio channel fully utilized. From the results obtained in urban areas, for the three scenarios TPA clearly outperforms the default scheme as well as another adaptive transmit power algorithm in terms of throughput (network capacity is maximized). Such findings call for the design of accurate policies for $802.11 \mathrm{P} / \mathrm{WAVE}$ standard which will adapt parameters based on the current and past situations and the current objective in order to tackle both the congestion and the fairness degradation problem when using an alternating access between $\mathrm{CCH}$ and $\mathrm{SCH}$.

\section{REFERENCES}

[1] N. Aschenbruck, R. Ernst, E. Gerhards-Padilla, and M. Schwamborn. Bonnmotion: a mobility scenario generation and analysis tool. In Proceedings of the 3rd International ICST Conference on Simulation Tools and Techniques, SIMUTools '10. ICST, 2010.

[2] J. Harri, F. Filali, C. Bonnet, and M. Fiore. Vanetmobisim: generating realistic mobility patterns for vanets. In Proceedings of the 3rd international workshop on Vehicular ad hoc networks, VANET '06, pages 96-97, New York, NY, USA, 2006. ACM.

[3] F. Karnadi, Z. H. Mo, and K.-C. Lan. Rapid generation of realistic mobility models for vanet. In Wireless Communications and Networking Conference, 200\%. IEEE WCNC 200\%., March 2007.

[4] K. Katsaros, M. Dianati, R. Tafazolli, and R. Kernchen. Clwpr - a novel cross-layer optimized position based routing protocol for vanets. In $V N C$ '11, 2011.

[5] V. Kawadia and P. Kumar. Principles and protocols for power control in wireless ad hoc networks. Selected
Areas in Communications, IEEE Journal on, jan. 2005.

[6] H. Menouar, M. Lenardi, and F. Filali. Movement prediction-based routing (mopr) concept for position-based routing in vehicular networks. In Vehicular Technology Conference, 2007. VTC-2007 Fall., Oct. 2007.

[7] G. Pallis, D. Katsaros, M. Dikaiakos, N. Loulloudes, and L. Tassiulas. On the structure and evolution of vehicular networks. In IEEE International Symposium on Modeling, Analysis Simulation of Computer and Telecommunication Systems, 2009. MASCOTS '09., 2009.

[8] D. B. Rawat, D. C. Popescu, G. Yan, and S. Olariu. Enhancing vanet performance by joint adaptation of transmission power and contention window size. IEEE Transactions on Parallel and Distributed Systems, 2011.

[9] P. Shankar, T. Nadeem, J. Rosca, and L. Iftode. Cars: Context-aware rate selection for vehicular networks. In IEEE International Conference on Network Protocols, 2008. ICNP 2008., Oct. 2008.

[10] A. Souza and A. L. Barros. An adaptive mechanism for access control in vanets. In ICN 2011: The Tenth International Conference on Networks, 2011.

[11] M. Torrent-Moreno, D. Jiang, and H. Hartenstein. Broadcast reception rates and effects of priority access in 802.11-based vehicular ad-hoc networks. In Proceedings of the 1st ACM international workshop on Vehicular ad hoc networks, VANET '04, New York, NY, USA, 2004. ACM.

[12] M. Torrent-Moreno, J. Mittag, P. Santi, and H. Hartenstein. Vehicle-to-vehicle communication: Fair transmit power control for safety-critical information. IEEE Transactions on Vehicular Technology, 58(7), 2009.

[13] M. Torrent-Moreno, P. Santi, and H. Hartenstein. Distributed fair transmit power adjustment for vehicular ad hoc networks. In Sensor and Ad Hoc Communications and Networks, 2006.

[14] X.-B. Wang, Y.-L. Yang, and J.-W. An. Multi-metric routing decisions in vanet. In Proceedings of the 2009 Eighth IEEE International Conference on Dependable, Autonomic and Secure Computing, DASC '09, Washington, DC, USA, 2009. IEEE Computer Society.

[15] F. Xue and P. R. Kumar. The number of neighbors needed for connectivity of wireless networks. Wirel. Netw., 10(2), March 2004.

[16] L. Yang, J. Guo, and Y. Wu. Channel adaptive one hop broadcasting for vanets. In 11th International IEEE Conference on Intelligent Transportation Systems, 2008. ITSC 2008., Oct. 2008. 